\title{
Argumentos para reconocer a los deportistas como trabajadores públicos civiles: más allá de la carrera dual
}

\section{Arguments for recognizing athletes as civil public workers: beyond dual career \\ Argumentos para reconhecer atletas como trabalhadores públicos civis: Além da dupla carreira}

\author{
Rodrigo Soto-Lagos ${ }^{a}$ (D), Mauro Navarrete ${ }^{b, *}$ (iD), Solange Freire ${ }^{c}$ (D), Juan Carlos López ${ }^{c}$ (iD, \\ Simón Martínez ${ }^{d}$ (D) , Jacinta Vélez ${ }^{\mathrm{a}}$ (D) , Ana Carolina Cortés ${ }^{\mathrm{a}}$ (D) Natalia Quijada $^{\mathrm{a}}$ (D)
}

Palabras clave

Deportistas;

Políticas públicas;

Trabajo;

Carrera dual.

\section{Keywords}

Atletes;

Public policies;

Work;

Dual career.

Palavras-chave

Atletas;

Políticas públicas;

Trabalho;

Carreira dupla.

\begin{abstract}
RESUMEN
El deporte es una actividad que se práctica de forma recreativa o profesional. Quienes lo hacen profesionalmente en ocasiones reciben aportes económicos y logran estudiar una carrera profesional, lo que se conoce como carrera dual. No obstante, hay quienes presentan esta actividad como su trabajo. El objetivo general del estudio fue analizar los argumentos de los deportistas chilenos para referirse a la práctica deportiva como una actividad laboral; el objetivo específico fue reflexionar críticamente sobre el desarrollo de la carrera dual en Chile. Para comprender estos argumentos, se realizó un estudio cualitativo por medio de entrevistas activas semiestructuradas a deportistas de alto rendimiento (DAR) y recreativos, olímpicos y paralímpicos. Los resultados muestran que los atletas demandan reconocimiento como trabajadores públicos por medio de tres argumentos: económico, subordinación y dependencia e identidad. Las conclusiones y discusiones sugieren debatir el reconocimiento de ellos y ellas como trabajadores públicos.
\end{abstract}

\section{ABSTRACT}

Sport is an activity practiced recreationally or professionally. Those who do it professionally occasions receive financial contributions and manage to study a professional career, which is known as a dual career. However, there are those who present this activity as their work. The objective of the study was to analyze the arguments of Chilean athletes to refer to sports as a work activity. The specific objective was to critically reflect on the development of the dual career in Chile To understand these arguments, a qualitative study was conducted through semi-structured active interviews with high performance athletes and recreational, Olympic and Paralympic athletes. The results show that athletes demand recognition as public workers through three arguments: economic, subordination and dependency, and identity. The conclusions and discussions suggest to debate their recognition as public workers.

\section{RESUMO}

O esporte é uma atividade praticada recreativa ou profissionalmente. Quem pratica profissionalmente às vezes recebe contribuições financeiras e consegue estudar uma carreira profissional, conhecida como carreira dupla. No entanto, há quem apresente essa atividade como trabalho. O objetivo do estudo foi analisar os argumentos de atletas chilenos para se referir ao esporte como atividade de trabalho. O objetivo específico foi refletir criticamente sobre o desenvolvimento da carreira dupla no Chile Para entender esses argumentos, foi realizado um estudo qualitativo, por meio de entrevistas ativas semiestruturadas com atletas de alto desempenho (DAR) e atletas recreativos, olímpicos e paralímpicos. Os resultados mostram que os atletas exigem reconhecimento como funcionários públicos por meio de três argumentos: econômico, subordinação e dependência e identidade. As conclusões e discussões sugerem debater seu reconhecimento como funcionários públicos.

aUniversidad Andrés Bello, Facultad de Educación y Ciencias Sociales. Viña del Mar, Chile.

bUniversidad de Chile, Facultad Ciencias Sociales. Santiago, Chile.

'Universidad Andres Bello, Facultad de Educación y Ciencias Sociales. Viña del Mar, Chile.

'Universidad Academia de Humanismo Cristiano, Facultad Ciencias Sociales. Santiago, Chile.

*Autor correspondente:

Mauro Navarrete.

E-mail: alonsonjz@gmail.com

Recibido el 23 de abril de 2020; aceptado el 24 de septiembre de 2020. 


\section{INTRODUCCIÓN}

En Latinoamérica, los Estudios Socioculturales del Deporte (ESD) (Alabarces, 1998, 2009; Guedes, 1997; Da Matta, 1982; Archetti, 1985), han desarrollado diferentes investigaciones que abren nuevos focos para estudiar el deporte en la sociedad. Este campo concibe los deportes como prácticas corporales construidas social e históricamente, que no portan valores ni esencias naturales. Esto quiere decir que las valoraciones sobre esta actividad adquieren sentido según los intereses de las personas o instituciones que en un determinado momento sociohistórico tienen la potestad de dirigirlo (Heinemann, 2001).

En coherencia con lo señalado, Giulianotti (2016) presenta al deporte como una "pseudopraxis" ya que, según su opinión, esta práctica contiene reglas que se asemejan a la competitividad brutal del mercado capitalista, pero que no representan al juego del deporte. En una línea similar, Coakley (2015) destaca que en la sociedad actúa el imaginario que señala que el trabajo duro y la disciplina estarían directamente relacionados con los logros deportivos. Así, la eficiencia, la racionalidad y la competencia se naturalizan como cualidades del deporte (Corriente y Montero, 2014) y, en efecto, se espera que también sean cualidades de los y las deportistas.

Respecto a esta práctica, existen personas que realizan deporte con regularidad y que incluso lo convierten en su dedicación, y son quienes en la medida en que se les reconocen sus habilidades, pueden ser contratados por clubes profesionales y así forjar una carrera profesional. No obstante, esto no ocurre de la misma manera con otras disciplinas deportivas ya que la regulación sobre el deporte profesional es diferente en cada país, en coherencia con las particularidades culturales de cada deporte.

\section{DEPORTE PROFESIONAL EN CHILE}

En Chile, desde el año 2007, la ley $\mathrm{N}^{\circ} 20.178$ comenzó a regular la relación laboral de los deportistas profesionales y trabajadores que desempeñan actividades conexas. A pesar de que este documento oficial se refiere a "deportistas", esta ley sólo aplica a los futbolistas que ejercen esta actividad como su profesión. Para remediar esta situación, el 22 de agosto de 2019 se ingresó un proyecto de ley que busca ampliar la definición de "deportes profesionales" a otras disciplinas deportivas, incluyendo el basquetbol y cualquier otra actividad afín (ver Boletín $\mathrm{N}^{\circ}$ 12.902-29). En la actualidad, esta propuesta se encuentra aún en trámite en el Congreso de la República, por lo que la dedicación profesional al deporte excluye de la regulación laboral a cualquier otra disciplina deportiva que no sea el futbol profesional.

Tomando en cuenta lo anterior, la mayoría de los deportistas que se dedican por completo a esta actividad no tienen la posibilidad de ser considerados trabajadores.
Esta situación ha sido considerada problemática en varios países del mundo, lo que ha llevado a generar acciones para apoyar a quienes se dedican al deporte como su actividad fundamental y que han optado por complementarla con el estudio o con el trabajo. Una de las propuestas que ha cobrado relevancia en el último tiempo ha sido la carrera dual (Stambulova y Wylleman, 2015).

\section{CARRERA DUAL}

El concepto de carrera dual (CD) se generó en la Unión Europea y se desarrolla en el documento denominado "Guidelines on Dual Careers of Athletes: Recommended Policy Actions in Support of Dual Careers in High-Performance Sport" (European Union, 2012). En él se señala que esta es una estrategia que busca que los y las atletas puedan conciliar el deporte con el trabajo o con el estudio. Uno de los argumentos que se destacan en este documento refiere que los atletas han reportado una posición de desventaja comparada con otros trabajadores en el mercado laboral, ya que la evidente priorización del deporte por sobre otra actividad, no les permite desarrollar, al mismo tiempo que los demás, las competencias requeridas en el mundo del trabajo. Además, se menciona que existen desafíos importantes respecto de las regulaciones y especificaciones legales frente al desarrollo de atletas jóvenes y discapacitados; también sobre el equilibrio entre estudio, trabajo y deporte; y frente a la retirada o término de la carrera deportiva.

En este tema, Debois et al. (2015) mencionan que existen factores que facilitan y limitan el curso de la vida de los y las atletas, dependiendo de la etapa de la carrera. También, Tekavc et al. (2015) reconocen diferentes factores psicosociales que influyen en el desarrollo de su doble carrera, tales como el estrés (Surujlal et al., 2013), el tiempo y la tensión que viven los deportistas para compatibilizar sus obligaciones deportivas y estudiantiles (Burlot et al., 2018), estilo de vida (MacCosham et al., 2015), la explotación de los cuerpos (Barker et al., 2014) y el apoyo que reciben (Knight et al., 2018). Todos estos aspectos podrían condicionar la mantención o el abandono del deporte (Hollings et al., 2014).

Por lo señalado, se ha expresado la necesidad de que los programas de carrera dual enfaticen la autonomía y la planificación profesional (Martin et al. 2014), la toma de decisiones (Miró et al., 2017) e incluso los aportes económicos o becas (Geiger, 2013; Harrison y Bukstein, 2014; Hopkinson et al., 2018; Richardson y McKenna, 2020).

En este mismo tema Pérez-Rivases et al. (2017) concluyen que la carrera dual es un proceso complejo producto de la preparación e inmersión tanto en el deporte como en el ámbito laboral. De hecho, Guirola Gómez et al. (2018), al analizar los facilitadores y barreras sobre este tema, destacan como obstáculo la no compensación económica hacia los deportistas. 
Asimismo, Brown (2015) menciona que, pese a que el deporte de alto rendimiento es un gran negocio, actualmente no existe claridad respecto del carácter del reconocimiento económico que reciben los y las deportistas.

El reciente estudio de Stambulova y Wylleman (2019) señalan que la investigación cuantitativa domina la producción de conocimiento en este campo, así como también ofrecen algunos desafíos para continuar estudiando este tema. Uno de ellos es desarrollar perspectivas de investigación que permitan considerar a los deportistas desde un punto de vista amplio, tomando experiencias del deporte y de otras dimensiones de sus vidas. Otro, señala que es preciso generar marcos de entendimiento e instrumentos teóricos adaptados culturalmente, para así interpretar los datos producidos de forma contextualizada.

\section{IDENTIDAD DEPORTIVA}

En coherencia con lo señalado, este trabajo dialoga con la investigación que se realiza en Europa respecto del discurso de la carrera deportiva desde la psicología del deporte (Stambulova, 2016a, b). Asimismo, se agregan nociones contextuales sobre Latinoamérica a través de la perspectiva denominada Psicología Social y Critica del Deporte (Soto-Lagos et al., 2020b) que considera la identidad como un aspecto importante para el desarrollo de la práctica deportiva.

El concepto de identidad en general y, la identidad deportiva (Tusak et al., 2005) en particular, se entiende en este estudio desde un punto de vista antiesencialista. Eso quiere decir que la identidad deportiva no es "algo" que está dentro de la persona, o como señala Koeneke (2018) una composición entre la identidad personal y la identidad social, sino que es una construcción cultural, material, social e históricamente situada (Soto-Lagos et al., 2020a). Más en coherencia con lo que señalan Brewer et al. (1993, en Martin et al., 2014) que entienden la identidad deportiva como el grado en que un individuo piensa y siento como un atleta.

Respecto del caso chileno, según el estudio de SotoLagos et al. (2020a), la identidad deportiva se construye considerando las condiciones socioculturales y materiales del país, las cuales están marcadas por lógicas y valores neoliberales. Este mismo estudio plantea que los valores con los que se promueve esta identidad en el país son el esfuerzo, la perseverancia, la garra y la pasión, los que pueden incluso

[...] invisibilizar precariedades y desigualdades en la vida de los deportistas y en el acceso a los bienes necesarios para desarrollar su labor de forma digna. En este punto, se sugiere desnaturalizar estas posiciones y discursos como esencialmente positivas ya que [...] en ocasiones éstas validan la precariedad, la desigualdad y las injusticias. (Soto-Lagos et al., 2020a, p. 11).
Según lo señalado, la identidad deportiva se encuentra condicionada por diversos factores, siendo el económico uno de gran relevancia entendiendo que para la dedicación al deporte además de motivación y talento, se requiere, sobre todo, apoyo económico para quien opta por esta actividad.

\section{APORTE PÚBLICO A LOS DEPORTISTAS EN CHILE}

Dado que la gran mayoría de los deportistas en Chile, salvo los futbolistas profesionales, no son considerados sujetos de derecho para contraer un contrato laboral por esta dedicación, el Estado ofrece apoyos económicos para quienes cumplen con ciertas condiciones. El principal instrumento de apoyo público es el Programa de Becas para Deportistas de Alto Rendimiento del Ministerio del Deporte, conocido como PRODDAR. Este programa se presenta como un incentivo o beca económica para los atletas y sus entrenadores, en función de resultados obtenidos en campeonatos internacionales. El beneficio se agrupa en siete niveles para los deportes individuales y en cuatro para deportes colectivos. Dentro de las diversas declaraciones que hace el programa, se destacan los beneficios y obligaciones.

Respecto a los beneficios, se señala que los favorecidos tienen derecho a recibir apoyo económico, asistencia técnico-metodológica, derecho a usar infraestructura pública disponible, atención de salud en el Centro de Alto Rendimiento (CAR), apoyo de las unidades de Ciencias del Deporte y asistencia social. En caso de requerir alimentación y residencia en el hotel del CAR, deben realizar una postulación específica para ello.

Frente a las obligaciones, el programa señala que los deportistas deben cumplir con su plan de entrenamiento, participar en eventos internacionales del ciclo olímpico y autorizar al Instituto Nacional de Deporte (IND) de manera gratuita e irrevocable a usar los derechos sobre su imagen, voz y nombre. Otra de las obligaciones refiere a no publicitar productos que estén en contra de los valores y principios del Olimpismo e informar al IND cuando convenga auspicios o patrocinios con la empresa privada.

En específico, el artículo 19 del documento que regula el PRODDAR señala que

[...] el deportista que reciba el incentivo económico mensual del Proddar deberá dedicarse de forma preferente a la actividad comprometida en el Plan de Entrenamiento" y que "será causal de suspensión o exclusión del Programa, conforme a lo contemplado en los artículos 47 y 48 de este Reglamento, realizar otras actividades que impidan objetivamente cumplir con su plan de entrenamientos, percibir, al mismo tiempo, un incentivo económico como entrenador o preparador físico de sí mismo u otros deportistas admitidos en el mismo programa, o realizar actividades como ser miembro o integrante de algún directorio de su Federación u otra organización menor, o ser parte 
de su personal administrativo. (Chile, 2015, Decreto 04/2015)

De acuerdo con lo señalado anteriormente, y en coherencia con el estudio de Soto-Lagos et al. (2020a), se aprecia que la realidad del deporte chileno requiere de miradas críticas para comprender y transformar los aspectos problemáticos. Un problema importante y contingente es que, hoy en día, los y las deportistas que quieran dedicarse a esta actividad como su trabajo, no pueden hacerlo ya que no existe la regulación jurídica ni administrativa para hacerlo. La única opción es recibir una beca a través del PRODDAR, lo que, en efecto no se ofrece como un salario mediado por un contrato de trabajo. Para avanzar en comprensión sobre este tema, el presente estudio se ha propuesto como objetivo general analizar los argumentos de los deportistas chilenos para referirse a la práctica deportiva como una actividad laboral y como especifico reflexionar críticamente sobre el desarrollo de la carrera dual en Chile.

\section{MÉTODOS}

\section{DISEÑO}

Tomando en cuenta el foco de esta investigación y la crítica realizada por Stambulova y Wylleman (2019) respecto a la hegemonía de las investigaciones con enfoque cuantitativo en el campo deportivo, el tipo de estudio realizado fue de carácter cualitativo (MorenoDoña et al., 2015), siguiendo las premisas de la Teoría Fundamentada (Glaser y Strauss, 1967). En coherencia con ello, la posición epistemológica de este estudio fue de carácter comprensivo/crítico (Ibáñez y Iñiguez, 1997; Iñiguez y Antaki, 1994), y ha buscado propiciar un diálogo con los estudios que señalan que para comprender ciertos fenómenos sociales se requiere considerar la voz de los actores y actrices que actúan en el campo deportivo (Fullagar, 2017; Smith y McGannon, 2018), en este caso a los y las deportistas.

\section{PARTICIPANTES.}

Los y las participantes, fueron seleccionados por medio de un muestreo teórico (Glaser y Strauss, 1967) conformando por cuatro categorías:

a) Deporte de Alto Rendimiento Convencional (DARC): Son quienes realizar deporte y compiten a nivel nacional e internacional en deportes olímpicos y no olímpicos;

b) Deporte de Alto Rendimiento Adaptado (DARA): Son quienes realizar deporte y compiten a nivel nacional e internacional en deportes bajo la modalidad adaptada o paralímpica;

c) Deporte Social/Recreativo Convencional (DSC): Son quienes realizar deporte con el objetivo de recrearse o hacer uso de su tiempo libre en modalidad convencional; d) Deporte Social/Recreativo Adaptado (DSCA): Son quienes realizar deporte con el objetivo de recrearse o hacer uso de su tiempo libre en modalidad adaptada.

Para resguardar su identidad y para asegurarnos que los relatos que aquí se presentan no pondrían en riesgo a los entrevistados, no se hizo referencia a las disciplinas deportivas y se usaron nombres de fantasía para citar las entrevistas. No obstante, sí es prudente mencionar que en la muestra: a) todos los deportistas se encuentran activos tanto competitiva como recreativamente. b) Los y las deportistas de alto rendimiento convencional y adaptado entrevistados han obtenido importantes logros para el país en torneos internacionales (Mundiales, Juegos Panamericanos, Juegos Olímpicos). c) Todos y todas quienes están en el alto rendimiento han tenido experiencia con el PRODDAR. d) Los y las deportistas sociales/recreativos son personas que practican deportes de forma constante hace más de dos años, lo que les otorga experiencia y conocimiento sobre el campo. e) El rango de edades de quienes entrevistamos fluctuó entre los 18 y 34 años.

\section{HERRAMIENTAS}

La herramienta de usada para producir los datos fue la entrevista activa semiestructurada (Sisto y Fardella, 2008). Éstas se llevaron a cabo entre el 1 de julio y el 31 de diciembre de 2018. Se seleccionaron diecisiete personas, 9 hombres y 8 mujeres, todos y todas de diferentes disciplinas y modalidades deportivas (Tabla 1) y se abordaron tres grandes temas. El primero, se vinculó con la historia personal y deportiva de la persona, en donde se abordaron temas tales como los valores construidos en esta actividad, las expectativas respecto al deporte, la educación y el futuro, los logros, su entorno significativo y los elementos motivacionales. El segundo, giró en torno la relación entre el deportista y los beneficios que recibía en tanto tal, con preguntas asociadas al reconocimiento de parte del Estado, la identidad deportiva y los mantenedores y obstaculizadores para su desempeño. El tercero, por su parte, se centró en las dimensiones sociales de la figura del deportista, tratando temas tales como el rol social y público de los y las atletas, la proyección de una familia y la producción de recursos económicos.

Cada entrevista duró entre 40 y 60 minutos. Al momento de solicitar autorización para grabar, se entregó un consentimiento informado con el que accedieron a participar voluntariamente del estudio. Consecutivamente, todas las entrevistas fueron transcritas siguiendo literalmente lo que cada persona mencionó en el diálogo. Se declara que algunas expresiones que sólo se usan en Chile fueron modificadas a un castellano neutro para que las citas sean entendidas por cualquier hablante de esta lengua. 
Tabla 1. Resumen de entrevistados.

\begin{tabular}{ccc}
\hline Nombre & Modalidad & Sexo \\
\hline Camilo & DARC & Hombre \\
Gonzalo & & Hombre \\
Javiera & & Mujer \\
Camila & & Mujer \\
Ximena & & Mujer \\
Carlos & & Hombre \\
Gustavo & & Hombre \\
Gabriel & DARA & Hombre \\
Diego & & Hombre \\
Jenny & & Mujer \\
Fernanda & & Mujer \\
Jaime & & Hombre \\
Katherine & DSC & Mujer \\
Daniel & & Hombre \\
Martín & & Hombre \\
Ema & & Mujer \\
Carolina & DSA & Mujer \\
\hline
\end{tabular}

Fuente: Elaboración Propia.

Tabla 2. Resultados de Investigación

\begin{tabular}{ll}
\hline \multicolumn{1}{c}{ Categorías } & \multicolumn{1}{c}{ Códigos } \\
\hline Económico & $\begin{array}{l}\text { Económicos } \\
\text { Sociales } \\
\text { Salud }\end{array}$ \\
Subordinación y Dependencia & $\begin{array}{l}\text { Rol del Estado } \\
\text { Producto del trabajo } \\
\text { Contrato de trabajo } \\
\text { Identidad }\end{array}$ \\
& Disputa noción de trabajo \\
& Diferenciación \\
\hline
\end{tabular}

Fuente: Elaboración Propia.

\section{PROCEDIMIENTO}

Para realizar el análisis, se construyó una unidad hermenéutica utilizando el software Atlas.ti 7.5. Se leyeron todas las entrevistas en reiteradas ocasiones y seguidamente se codificaron teniendo en cuenta los argumentos de los deportistas para construir el deporte como un trabajo.

\section{ANÁLISIS DE DATOS}

Para realizar el análisis se construyó una unidad hermenéutica utilizando el software de análisis de datos cualitativos Atlas.ti 7.5. Seguidamente, las entrevistas fueron leídas en varias ocasiones. Posteriormente, se codificaron teniendo en cuenta los argumentos que utilizaron los y las deportistas para referirse al deporte como su trabajo.
Luego de lo anterior, se efectuó una codificación abierta con las categorías emergentes, tal como propone la Grounded Theory (Gibbs, 2012; Glaser y Strauss, 1967; Strauss y Corbin, 2002). En el paso siguiente, se realizó una codificación axial para reunir las categorías emergentes en familias de categorías. El último paso consistió en realizar la codificación selectiva que permitió llevar a cabo el trabajo de interpretación de la información producida (Moreno-Doña et al., 2015).

A partir de lo anterior, se obtuvieron nueve códigos, los que se agruparon en tres categorías (Tabla 2) que señalan cuáles son los argumentos que presentan los y las deportistas para referirse a la práctica deportiva como una actividad laboral. Se aclara que el nombre de los códigos y categorías fueron asignados por el equipo investigador para facilitar el entendimiento de los resultados.

Para la validación de la investigación se tomaron los criterios de transferibilidad y representatividad propuestos por Lincoln y Guba (1985) y reelaborados por Sisto (2008). Estas nociones permiten expresar que los resultados de esta investigación podrían transferirse a otros contextos y potencialmente, dada la representatividad de la información, los dichos de nuestros entrevistados podrían representar las experiencias, sentires, emociones y opiniones de otros deportistas tanto de chile como de algún otro lugar del mundo que esté sujeto a condiciones similares. Asimismo, para aportar con credibilidad a los resultados, el borrador de este escrito fue devuelto a los participantes, quienes, a su vez, hicieron sus comentarios sobre la información entregada.

Se menciona también que este estudio fue aprobado por la comisión de ética de la Facultad de Educación y Ciencias Sociales de la Universidad Andrés Bello de Chile, organismo que asegura haber cumplido todos los resguardos éticos para el trabajo de investigación con seres humanos.

\section{RESULTADOS}

Luego del análisis realizado, se construyeron tres categorías que sirven para explicar los argumentos de los y las atletas con relación a reconocer su actividad como un trabajo público. Estos son: económico, subordinación y dependencia e identidad.

\section{ECONÓMICO}

Respecto a esta dimensión, los y las atletas enuncian aspectos económicos, sociales y de salud que son entendidos como situaciones que deberían ser resueltas para asegurar mejores condiciones para su desempeño como atletas. Refieren, específicamente, que acceder a un contrato de trabajo les permitiera tener un salario, cotizaciones previsionales, acceder a créditos bancarios (hipotecarios) y asegurar el acceso a una salud de relativa calidad. Estas reivindicaciones, desde la voz de los atletas, 
son avances que se deberían considerar para mejorar el deporte en el país.

Acá eres una persona que hace deporte. Hay un deporte profesional no más, hay dos, el básquet que no sé cómo se sostiene, y está el fútbol. El resto sobrevive. No [puedes] hablar de cotizar en una AFP siendo deportista, olvídate. No [puedes] hablar de un futuro más allá siendo deportista, porque esto eso es un tema de becas, no un sueldo [...] O sea, por mí que sea un sueldo. Porque [tienes] que pensar que ahora te [estás] dedicando a tu deporte, pero el deporte va a ser pan para hoy, hambre para mañana. (Gonzalo. DARC).

En la cita, el entrevistado señala que en Chile los atletas son "personas que hacen deporte" lo que pragmáticamente expresa que no existe la categoría "deportista" más allá de deportes profesionales como el futbol o el basquetbol, en sintonía con lo señalado en la ley N²0.178. Con la expresión "el resto sobrevive" se construye una inferioridad de las disciplinas deportivas respecto del futbol profesional lo que no les permitiría "hablar de un futuro más allá siendo deportista". Eso podría implicar que las condiciones materiales actuales no permiten a quienes se dedican a esto, proyectarse hacia el futuro.

Seguidamente, para enfrentar lo dicho, el deportista declara que ellos y ellas deberían tener un sueldo y no una beca, como es hoy. Para fortalecer esta idea enuncia la metáfora "pan para hoy, hambre para mañana" la que coloquialmente se refiere a las personas que gastan sus recursos en el presente sin proyectar las eventualidades del futuro. En el contexto deportivo, la expresión sugeriría que la dedicación exclusiva a esta actividad no les permitiría imaginar seguridades económicas en el futuro.

En relación con lo mencionado, otra deportista sugiere tener "seguro médico" y "sueldo base" ya que ambos permitirían que los y las atletas tengan las condiciones mínimas de dignidad laboral.

Creo que los deportistas deberían tener seguro médico, deberían tener un sueldo base y deberían tener el acceso a poder desarrollar el deporte de la manera más grata posible. (Camila. DARC).

En este mismo plano, existe una interpelación a quienes toman decisiones para que construyan criterios que definan a los deportistas de Alto Rendimiento.

Pongan todos los criterios que quieran para considerar quien es de alto rendimiento, pero el que cumpla con esos criterios debería tener un sueldo, debería tener imposiciones, debería tener salud, debería tener como pedirle un crédito hipotecario a un banco, como tienen todos los trabajadores que tienen un sueldo en Chile. (Fernanda. DARA).

En la cita, la entrevistada interpela a quienes tienen el poder y la responsabilidad de tomar decisiones para que "pongan los criterios que quieran" para reconocer a los atletas de alto rendimiento. Sin embargo, cumplidos éstos, debería haber un sueldo, imposiciones, salud y la opción de acceder a créditos hipotecarios "[...] como tienen todos los trabajadores que tienen un sueldo el Chile", posicionando a los atletas como trabajadores del país, demandando igualdad de derechos como los de un trabajador convencional.

\section{SUBORDINACIÓN Y DEPENDENCIA}

Cuando los y las deportistas refieren a su labor, expresan que para ejercer su trabajo deben subordinarse a instituciones, equipos, entrenadores y, además, deben cumplir con sus horarios de entrenamiento. Asimismo, se aprecia que los y las deportistas deben mantener una relación dependiente hacia las autoridades de la disciplina, lo que expresa una radical contradicción entre las exigencias y beneficios ofrecidos hacia ellos y ellas.

Frente a este tema, una entrevistada expresa que el Estado debería garantizar el bienestar de los deportistas, argumentando que, al estar representando al país, deberían darse garantías desde esta entidad.

[...] yo creo que el Estado debería ser garante del bienestar del deportista porque está representando un país, está representando una entidad. (Camila. DARC).

En esta categoría, se plantea que al igual que cualquier otro trabajador los deportistas producen algo. Este fruto de su trabajo está asociado al estatus y a la representación de un país, generando una analogía entre su actividad con la de un embajador.

[...] si lo vemos por ese lado un deportista entrena y compite para él y para dejar como en alto el nombre de Chile [...] siento que se podría considerar como un trabajo, pero sería como un trabajador público, como algo para el país, sería para darle más estatus al país, para dejarle un nombre en alto, quizás como un tipo de embajador. (Martín. DSC).

Desde la cita se desprende que el producto del trabajo de los y las atletas es un bien social, expresado como "más estatus para el país", lo que otorga la fuerza discursiva para expresar que la labor de los y las deportistas podría ser considerado como un trabajo público. Esta idea se refuerza con la expresión "quizás como un tipo de embajador", ya que la labor que realizan estos profesionales es la de representar al país frente a otras naciones, algo similar a lo que hace un deportista.

Respecto a los apoyos que se reciben de parte del Estado, el PRODDAR dicta las pautas respecto de quien puede recibir este beneficio. Frente a este dispositivo público, un deportista señala lo siguiente:

A partir del año 20XX [...] ya me pude sustentar un poco más y hace poco me [expulsaron] por temas de que netamente tenía que hacer la práctica profesional [...] Por ende, no podía entrenar. EI PRODDAR es un contrato, y los contratos cuando una de las partes no 
hace bien, no cumple los términos, se corta. $Y$ eso, es así de cruel. (Gonzalo. DARC).

En el extracto, el deportista señala que fue beneficiario de PRODDAR, el cual se construye como un apoyo, como algo que sirve "para sustentar un poco" la vida y la carrera deportiva. Seguidamente, menciona que lo expulsaron por no asistir a los entrenamientos ya que decidió terminar su práctica profesional, lo que impedía compatibilizar ambas actividades. Por otro lado, el deportista enuncia que el PRODDAR es un contrato y que, si una de las partes no cumple con lo declarado en el documento, "se corta".

Desde la experiencia del deportista, haber sido "echado" del programa por decidir terminar su formación profesional, se vivió como algo "cruel" ya que, desde esta forma de concebir el contrato, él no habría "cumplido con los términos" señalados en éste.

\section{IDENTIDAD DEPORTIVA}

A lo largo de todas las entrevistas, los deportistas mencionan que la dedicación a esta actividad los interpela como personas y, en concreto, se convierte en una dimensión de su identidad. Este argumento permite distinguir, además, los valores que construyen sobre sí mismos respecto de la labor que realizan. Al respecto, una de las entrevistadas, señala:

[...] un deportista [...] tiene que ser alguien que es súper disciplinado, que hace todos los sacrificios del mundo como seguir dietas, o madrugar, o hacer los entrenamientos, o esos momentos en que estás cansado y dices; "no, ya no puedo más". Bueno, obviamente los deportistas olímpicos dicen: "Sí, sí, a pesar de que no puedo más, tengo que seguir". (Claudia. DARC).

En el fragmento, la entrevistada a través del auto diálogo presentado, declara que las cualidades de los deportistas son la disciplina y el sacrificio y, con la expresión "[...] los deportistas olímpicos dicen: "Sí, sí, a pesar de que no puedo más, tengo que seguir" construye una identidad particular a estos sujetos. En este sentido, los valores construidos a partir de la experiencia en el deporte se presentan como una parte fundamental de la identidad de los y las deportistas. Desde su voz, estas características cumplen la función de distinguirlos de la población no deportista, en la medida en que las vivencias del campo deportivo cobran más valor al trasladarse a otros espacios sociales.

Con este argumento, los deportistas disputan la noción de trabajo a partir de la particularidad de la actividad que realizan. Para hacerlo, los entrevistados refieren a la alta especialización que desarrollan para dedicarse a esta actividad, ya que estos deben cumplir un ideal físico y psicológico particular. Esta especialización les permite competir a una alta intensidad y conseguir resultados positivos, lo que los diferencia de otras actividades y trabajos.
[...] un deportista de alto rendimiento, si tú lo ves desde ese punto de vista, tiene que dormir cierta cantidad de horas, tiene que comer como se debe, a las horas que se debe, comida especial, proteínas qué sé yo, y además programarse con sus entrenamientos durante el día, o lo que tenga que hacer [¿entiendes?] Entonces, al final, tal vez es hasta más que un trabajo. (Katherine. DSC).

Además, los entrevistados construyen una diferenciación entre quienes se dedican al alto rendimiento y al deporte social, la que expresa un rol diferente en la sociedad, que dependería de la cantidad de horas dedicadas a esta práctica.

[...] yo creo que los atletas deberían ser considerados traba, como trabajadores porque es distinto una persona que haga deporte a un atleta. Atleta es el que entrena todos los días, el que es atleta [...] compite en deporte de alto rendimiento. Deportista es el que hace ejercicio tres veces a la semana, de lunes a viernes, [...] no es tan competitivo. Pero claro, los atletas deberían verse como trabajadores y debería haber un auspicio, un patrocinio, algo. (Gustavo. DARC).

A partir de lo anterior, serían atletas los que se dedican a entrenar para competir y serían deportistas, quienes entrenan por diversión, ocio o recreación. Con esta expresión, se presenta una identidad concreta que, tal como se ha desarrollado, argumenta que los deportistas reconocen una particularidad en la actividad que realizan.

\section{DISCUSIÓN}

Luego del análisis realizado, este estudio señala que tanto los deportistas de elite como atletas recreativos entrevistados, consideran que la dedicación al alto rendimiento debe ser considerado un trabajo. En Chile, según la Ley N²0.178, sólo el fútbol profesional masculino tiene esas cualidades, por lo que los deportistas de otras disciplinas no son tratados como trabajadores a pesar de que reciben apoyos económicos mensuales, cumplen horarios, deben lograr metas, obedecen jerarquías, representan a una institución y firman un contrato para recibir el aporte económico que, administrativamente, es presentado como una beca. Asimismo, la labor que realizan, dado las altas cargas de entrenamiento y dedicación, se presenta como un trabajo de alta especialización, muy similar a cualquier otra profesión que exige un perfeccionamiento constante.

Los argumentos presentados fueron organizados a partir de lo económico ya que, desde este plano, incluyendo aspectos sociales y de salud, se señala que la forma actual de apoyo a los atletas podría configurarse como una barrera para el desarrollo deportivo (Guirola Gómez et al., 2018). Este emplazamiento podría complementarse con las ideas de Burlot et al. (2018), en la medida que al no mediar un contrato de trabajo por el dinero que reciben los atletas mes a mes, podría presentarse un estado de tensión permanente 
que generaría efectos negativos en el bienestar (MacCosham et al., 2015).

En este punto, si bien es posible señalar que algunos atletas de alto rendimiento han optado por el desarrollo de una carrera dual (European Union, 2012; Stambulova y Wylleman, 2015), los datos señalan que no existe un sano equilibrio entre ambas actividades ya que cuando corresponde dedicar más tiempo al estudio, el PRODDAR se acaba por no cumplir con el contrato. Esto es coherente con lo señalado por Surujlal et al. (2013) y Burlot et al. (2018) quienes hacen visible el estrés y la tensión que viven los deportistas que intentan compatibilizar obligaciones deportivas y estudiantiles.

Otra dimensión de su argumento fue la subordinación y dependencia, que menciona que los y las atletas deben obedecer las jerarquías propias de un trabajo, pero en el campo deportivo. En sus palabras, cumplir con horarios de entrenamiento, subordinarse a las decisiones de entrenadores y dirigentes, organizar su vida para procurar obtener resultados deportivos y entregar sus derechos de imagen al Estado, se vive como la firma de un contrato de trabajo. No obstante, este contrato no promueve la autonomía y planificación profesional (Martin et al., 2014) ni la toma de decisiones (Miró et al., 2017) por lo que la forma de promover el deporte de alto rendimiento en Chile no favorece el desarrollo deportivo.

Otra de las cualidades presentadas fue la referida a la identidad deportiva. Asumimos las propuestas de Stambulova (2016a, b) que sugiere ir más allá del individuo en este tema, lo que permite señalar que los y las atletas experiencian el vínculo al PRODDAR como el acto de firmar un contrato de trabajo. Este hecho simbólico permea la identidad deportiva y amplia el grado en que un individuo se piensa y siente como un atleta (Brewer et al., 1993, en Martin et al., 2014), integrando a la identidad deportiva la dimensión de trabajadores.

Esto último disputa la noción de trabajo señalado en la Ley 20.178, ya que la alta especialización que necesitan desarrollar los deportistas de alto rendimiento para dedicarse a esta actividad, los convierte en trabajadores de alta calificación.

En general, los resultados también son coherentes con lo señalado por Pérez-Rivases et al. (2017) respecto a que la carrera dual es un proceso complejo. No obstante, los datos proponen una dimensión que va más allá de las cualidades psicológicas individuales propuestas por algunos estudios (Surujlal et al., 2013; Burlot et al., 2018; MacCosham et al., 2015; Knight et al., 2018; Hollings et al., 2014) y se podría manifestar que en Chile no se estaría desarrollando una buena práctica de carrera dual que facilite el complemento entre el estudio o el trabajo con la dedicación al deporte. Específicamente, cuando un deportista declaró que por decidir terminar su carrera profesional fue expulsado del programa, ofrece información para expresar que en el país esta modalidad reproduce la desigualdad y la inequidad en el acceso al estudio, al trabajo y/o al deporte ya que, según su relato, no se podría compatibilizar el deporte con una carrera profesional.

En esta línea, las experiencias relatadas por los y las deportistas cuestionan las implicancias económicas y sociales la noción de carrera dual. De esta manera, este estudio puede ser un aporte para generar investigación comparada respecto a esta temática, ya que al menos desde la experiencia chilena, no se logra compatibilizar fácilmente la formación profesional con la carrera deportiva.

\section{CONCLUSIÓN}

Esta investigación es un aporte a la discusión propuesta por Stambulova y Wylleman (2019) respecto a la carrera dual. Específicamente, suma la voz de deportistas chilenos, en sintonía con la invitación de Fullagar (2017) y Smith y McGannon (2018).

Lo anterior, permite afirmar que el deporte de alto rendimiento opera con una lógica extractivista en cuanto al reconocimiento de los deportistas como trabajadores. En el campo deportivo, entrenadores, gerentes, profesionales, entre otros roles, tienen un salario gracias a que existen los deportistas quienes, paradójicamente, no tienen contratos y no son reconocidos como trabajadores. Esto, se complementa con el estudio de Barker et al. (2014) quienes mencionan que es pertinente incluir una lógica de la sustentabilidad en el campo deportivo.

A partir de lo anterior, se sugiere que, para validar a los deportistas de alto rendimiento como trabajadores, es necesario cambiar la figura administrativa de beca por la de salario mediado por un contrato. Eso implicaría que las instituciones públicas y privadas inmersas en el deporte, deben asumir responsabilidades para con los sujetos que les permiten existir y presumir de logros deportivos nacionales o empresariales.

Asimismo, las entrevistas sugieren que sean considerados trabajadores públicos ya que el ente interpelado ha sido el Estado, quien en la actualidad ofrece el apoyo económico a través del PRODDAR. En este sentido, los autores de este estudio sugerimos que, en caso de ser considerados funcionarios públicos, deberían ser civiles y no militares. Si llegasen a ser considerados parte de las Fuerzas Armadas, se les estaría negando el derecho de organización y sindicalización para enfrentar democráticamente a las autoridades que toman decisiones que los y las afectan. En concreto, no podrían realizar una negociación colectiva ya que los militares deben someterse y asegurar la sumisión a las jerarquías establecidas en dichas instituciones, lo que despotencia la libertad y la capacidad de transformar el campo deportivo desde la voz de ellos mismos en tanto trabajadores.

En cuanto a las limitaciones de la presente investigación, se identifican tres puntos que podrían servir para desarrollarlos en otros estudios. El primero refiere a que no fueron incluidos futbolistas profesionales 
ya que, el relato de ellos podría aportar con experiencias, tensiones, problemas y sugerencias para pensar a los otros deportes como una actividad laboral. La segunda limitación, refiere al hecho de haber entrevistado sólo a una deportista de modalidad recreativa/social adaptada, lo que podría dar cuenta de que para personas en situación de discapacidad existe más posibilidades de realizar deporte en alto rendimiento que en la modalidad recreativa. La última limitación es que los resultados ofrecen una mirada sólo de Chile respecto a considerar a los deportistas como trabajadores, por ello instamos a realizar más estudios sobre esta temática para aclarar si esto es sólo un tema expresado en el país o si son temas transversales en el campo deportivo global.

\section{FINANCIAMENTO}

Fondo Jorge Millas, Universidad Andres Bello.

\section{CONFLICTO DE INTERÉS} interesse.

Os autores declaram não haver conflitos de

\section{REFERENCIAS}

Alabarces P. ¿De qué hablamos cuando hablamos de deporte? Revista Nueva Sociedad. 1998;154:74-86.

Alabarces P. El deporte en América Latina. Revista Razón y Palabra. 2009; 69.

Archetti E. Fútbol y ethos en monografías e informes de investigación. Argentina: FLACSO. 1985. (Serie Investigaciones).

Barker D, Barker-Ruchti N, Wals A, Tinning R. High performance sport and sustainability: a contradiction of terms? Reflective Pract. 2014;15(1):1-11. http://dx.doi.org/10.1 080/14623943.2013.868799.

Brown S. Moving elite athletes forward: examining the status of secondary school elite athlete programmes and available post-school options. Phys Educ Sport Pedagogy. 2015;20(4):442-58. http://dx.doi.org/10.1080/17408989 .2014 .882890 .

Burlot F, Richard R, Joncheray H. The life of high-level athletes: the challenge of high performance against the time constraint. Int Rev Sociol Sport. 2018;53(2):234-49. http:// dx.doi.org/10.1177/1012690216647196.

Chile. Decreto 4/2015. Deroga decreto № 29, de 2012, del Ministerio Secretaría General de Gobierno, y aprueba nuevo reglamento del programa de becas para deportistas de alto rendimiento del Ministerio del Deporte. Biblioteca del Congreso Nacional de Chile (BCN); 11 nov. 2015. Disponible en: http://bcn.cl/2geiu.

Coakley J. Sports in society, issues and controversies. New York: Mc Graw Hill Education; 2015.

Corriente F, Montero J. Citius, Altius, Fortius: el libro negro del deporte. Rosario, Argentina: Lazo Ediciones; 2014.

Da Matta R. Esporte na Sociedade: un ensaio sobre o futebol brasileiro. In: DaMatta R, editor. Universo do futebol: esporte e sociedade brasileira. Rio de Janeiro: Pinakotheke; 1982. p. 19-42
Debois N, Ledon A, Wylleman P. A lifespan perspective on the dual career of elite male athletes. Psychol Sport Exerc. 2015;21:15-26. http://dx.doi.org/10.1016/j. psychsport.2014.07.011.

European Union. EU guidelines on dual careers of athletes: recommended policy actions in support of dual careers in high-performance sport [Internet]. Brussels; 2012 [citado 2020 abril 15]. Disponible en: https://ec.europa.eu/assets/ eac/sport/library/documents/dual-career-guidelinesfinal_en.pdf

Fullagar S. Post-qualitative inquiry and the new materialist turn: Implications for sport, health and physical culture research. Qual Res Sport Exerc Health. 2017;9(2):247-57. http://dx.doi.org/10.1080/2159676X.2016.1273896.

Geiger N. Intercollegiate athletics in Canada and the United States: differences in access, quality, and funding. Coll Q [Internet]. 2013;16(3):1-17. [citado 2020 abril 15]. Disponible en: https://eric.ed.gov/?ft=on\&q=support+at hletes\&ff1=subFinancial+Support\&id=EJ1017978

Gibbs G. El análisis de datos cualitativos en investigación cualitativa. Madrid: Morata; 2012.

Giulianotti R. Sport a critical sociology. Cambridge: Polity Press; 2016.

Glaser B, Strauss A. The discovery of grounded theory: strategies for qualitative research. Chicago: Aldine; 1967.

Guedes S. O futebol brasileiro: instituição zero [dissertação]. Rio de Janeiro: Universidade Federal do Rio de Janeiro; 1997.

Guirola Gómez I, Torregrosa M, Ramis Y, Jaenes JC. Remando contracorriente: facilitadores y barreras para compaginar el deporte y los estudios. Rev Andal Med Deporte. 2018;11(1):12-7. http://dx.doi.org/10.1016/j. ramd.2016.08.002.

Harrison C, Bukstein S. Canaries in the goal mines: a timely analysis of college athletics and the role of studentathletes. J Intercoll Sport. 2014;7(2):109-19. http://dx.doi. org/10.1123/jis.2014-0133.

Heinemann K. Los valores del deporte: una perspectiva sociológica. Rev Apunts Educ Fis Deporte. 2001;64:17-25.

Hollings S, Mallett C, Hume P. The transition from elite junior track-and-field athlete to successful senior athlete: why some do, why others don't. Int J Sports Sci Coaching. 2014;9(3):457-71. http://dx.doi.org/10.1260/17479541.9.3.457.

Hopkinson M, Smolianov P, Dion S, Norberg J. Comparing practices of US volleyball systems against a global model for integrated development of mass and high performance sport. ICHPER-SD J Res. 2018;9(2):11.

Ibáñez T, Iñiguez L, comp. Critical social psychology. London: SAGE; 1997. http://dx.doi.org/10.4135/9781446279199.

Iñiguez L, Antaki C. El análisis del discurso en psicología social. Bol Psicol. 1994;44:57-75.

Knight C, Harwood C, Sellars P. Supporting adolescent athletes' dual careers: the role of an athlete's social support network. Psychol Sport Exerc. 2018;38:137-47. http:// dx.doi.org/10.1016/j.psychsport.2018.06.007. 
Koeneke H. Eventos deportivos como lentes para abordar desarrollo urbano, identidad y relaciones de poder. Bol Cient Sapiens Res. 2018;8(2):3-9.

Lincoln E, Guba Y. Naturalistic inquiry. Beverly Hills: Sage; 1985. http://dx.doi.org/10.1016/0147-1767(85)90062-8.

MacCosham B, Patry P, Beswick C, Gravelle F. Leisure lifestyle and dropout: exploring the experience of amateur athletes in competitive sport. Int J Sport Manag Recreat Tour. 2015;20:20-39. http://dx.doi.org/10.5199/ijsmart-1791$874 \mathrm{X}-20 \mathrm{~b}$

Martin L, Fogarty G, Albion M. Changes in athletic identity and life satisfaction of elite athletes as a function of retirement status. J Appl Sport Psychol. 2014;26(1):96-110. http:// dx.doi.org/10.1080/10413200.2013.798371.

Miró S, Torregrosa M, Reguela S, Perez A, Ramis Y. Competencias para la planificación de la carrera dual de deportistas de alto rendimiento. Rev Psicol Deporte. 2017;26:51-6.

Moreno-Doña A, Rivera-García E, Trigueros-Cervantes C. La educación física en Chile: un análisis de las creencias del profesorado de la enseñanza primaria y secundaria. Movimento. 2015;20(esp):81-96. http:// dx.doi.org/10.22456/1982-8918.48098.

Pérez-Rivases S, Torregrossa M, Pallares S, Viladrich C, Regüela $\mathrm{S}$. Seguimiento de la transición a la universidad en mujeres deportistas de alto rendimiento. Rev Psicol Deporte. 2017;26(3):102-7.

Richardson J, McKenna S. An exploration of career sustainability in and after professional sport. J Vocat Behav. 2020;117:103314. http://dx.doi.org/10.1016/j. jvb.2019.06.002.

Sisto V, Fardella C. Narrándose en la flexibilidad: un análisis narrativo discursivo de la identidad en tiempos de flexibilidad laboral. Rev Psicol. 2008;17(2):59-80.

Sisto V. La investigación como una aventura de producción dialógica: la relación con el otro y los criterios de validación en la metodología cualitativa contemporánea. Psicoperspectivas. 2008;7(1):114-36.

Smith B, McGannon K. Developing rigor in qualitative research: problems and opportunities within sport and exercise psychology. Int Rev Sport Exerc Psychol. 2018;11(1):10121. http://dx.doi.org/10.1080/1750984X.2017.1317357.
Soto-Lagos, R., Navarrete-Jerez, M., Freire-Arancibia, S. y Cortés-Varas, A. Identidad deportiva en Chile: Un proceso sociocultural y material marcado por el Estado y el Neoliberalismo. Revista Movimento. 2020a; Porto Alegre, v. 26. https://doi.org/10.22456/1982-8918.100538.

Soto-Lagos R, Pozo-Gómez L, Romero Alvarez P. Hacia una psicología social y critica del deporte. Quad Psicol. 2020b;22(3):e1558. http://dx.doi.org/10.5565/rev/ qpsicologia.1558.

Stambulova N, Wylleman P. Dual career development and transitions. Psychol Sport Exerc. 2015;21:1-3. http://dx.doi. org/10.1016/j.psychsport.2015.05.003.

Stambulova N, Wylleman P. Psychology of athletes' dual careers: a state-of-the-art critical review of the European discourse. Psychol Sport Exerc. 2019;42:74-88. http:// dx.doi.org/10.1016/j.psychsport.2018.11.013.

Stambulova N. Athletes' transitions in sport and life: positioning new research trends within existing system of athlete career knowledge. In: Schinke RJ, McGannon K, Smith B, editors. The Routledge international handbook of sport psychology. New York: Routledge; 2016b. p. 519-35.

Stambulova N. Theoretical developments in career transition research: contributions of European sport psychology. In: Raab M, Wylleman P, Seiler R, Elbe A, Hatzigeorgiadis $A$, editors. Sport and exercise psychology research: from theory to practice. London: Elsevier; 2016a. p. 251-69. http://dx.doi.org/10.1016/B978-0-12-803634-1.00012-1.

Strauss A, Corbin J. Bases de la investigación cualitativa: técnicas y procedimientos para desarrollar la teoría fundada. Medellín: Editorial Universidad de Antioquia; 2002.

Surujlal J, Van Zyl Y, Nolan V. Perceived stress and coping skills of university student-athletes and the relationship with life satisfaction. Afr J Phys Health Educ Recreat Dance. 2013;19:1047-59.

Tekavc J, Wylleman P, Cecic-Erpic S. Perceptions of dual career development among elite level swimmers and basketball players. Psychol Sport Exerc. 2015;21:27-41. http://dx.doi. org/10.1016/j.psychsport.2015.03.002.

Tusak M, Faganel M, Bednarij J. Is athletic identity an important motivator? Int J Sport Psychol. 2005;36(1):39-49. 